\title{
Chemoprevention using folic acid for dysplastic lesions of the larynx
}

\author{
MASSIMO MESOLELLA $^{1}$, MAURIZIO IENGO ${ }^{1}$, DOMENICO TESTA ${ }^{2}$, \\ FILIPPO RICCIARDIELLO ${ }^{1}$ and BRIGIDA IORIO ${ }^{1}$
}

\footnotetext{
${ }^{1}$ Department of Neuroscience, Reproductive and Odontostomatologic Science, ENT Division, University 'Federico II'; ${ }^{2}$ Department of Anesthesiology, Surgery and Emergency Science, University 'Luigi Vanvitelli', I-80122 Naples, Italy
}

Received May 16, 2017; Accepted July 26, 2017

DOI: $10.3892 / \mathrm{mco} .2017 .1424$

\begin{abstract}
Folate deficiency may be directly associated with carcinogenesis. Folate supplementation may reduce the risk of progression in a mucosa that is already genetically altered. The aim of the present study was to determine the role of folic acid in the treatment of mild and moderate laryngeal dysplasia recurring after surgery. The data obtained by laryngoscopy, measurement of serum folate levels and functional vocal assessment with the GIRBAS scale and VHI questionnaire were compared in patients who had received folic acid with a homogeneous group of patients with dysplasia who had not undergone any therapy. A total of 24 patients suffering from recurrence of mild or moderate laryngeal dysplasia $(n=12$, group A) were treated with $400 \mathrm{mg}$ folic acid per os, once daily for 6 months. The patients in group $B(n=12)$ received no treatment and were used as the control group. In group A, $7(58 \%)$ patients exhibited a complete response, with clinically evident regression of leukoplakia, 3 (25\%) displayed a partial decrease in the lesions with reduced volume of the area involved, whereas 2 patients exhibited no change. As regards patients in control group B, $8(67 \%)$ exhibited no change, in 1 case there was a spontaneous regression, and in $3(25 \%)$ there was disease progression with suspected malignant transformation, leading to repeat surgery. Therefore, folate deficiency may be considered to be a factor predisposing to precancerous lesions, and dietary folate supplementation may prevent and reduce the emergence of cancer.
\end{abstract}

Correspondence to: Dr Massimo Mesolella or Dr Brigida Iorio, Department of Neuroscience, Reproductive and Odontostomatologic Science, ENT Division, University 'Federico II', 5 Via S. Pansini, I-80122 Naples, Italy

E-mail: massimo.mesolella@tin.it

E-mail: brigida.iorio82@gmail.com

Key words: laryngeal dysplasia, folic acid, chemoprevention

\section{Introduction}

Carcinogenesis is a multistep process including genetic alterations that develop over time, and it is associated with metabolic alterations that may promote neoplastic progression (1).

Metabolic processes that use folates interfere with the synthesis, repair and methylation of DNA, as they are involved in the synthesis of purine and of pyrimidine bases and serve as methyl donors in the methionine cycle (2).

Folate deficiency encourages the assimilation of uracil in the DNA, which causes a genomic instability characterized by the formation of single and double breaks in the DNA filaments, chromosomal breakages and the formation of micronuclei (2).

It is obvious, therefore, that folate deficiency may be directly implicated in promoting carcinogenesis $(3,4)$. The chronological succession of certain types of metaplasia of the laryngeal epithelium with laryngeal cancer may identify a group of lesions that are defined as 'precancerous' (5).

Several events that take place during the transformation of precancerous lesions to cancer may be controlled and even reversed by removing the possible trigger factors (e.g., discontinuing smoking and following a balanced diet) and by using carcinogenesis inhibitors (chemoprevention) (6). Chemoprevention is the use of chemical or natural substances to reverse, suppress or prevent conversion of precancerous to invasive lesions (7). At this time, chemoprevention may be defined as the use of agents to prevent the appearance of precancerous lesions, or delay or reverse progression from precancerous to cancerous lesions. The multistep theory of carcinogenesis (accumulation of genotypic or phenotypic alterations) has further highlighted the importance of chemoprevention in arresting these processes, as it may be sufficient to prevent the development of cancer $(8,9)$.

Folate supplementation, alone or in combination with other chemopreventive agents, may reduce the risk of progression in a mucosa that is already genetically altered, particularly in patients with folate deficiency. This is important, as laryngeal leukoplakia tends to recur, often with a histological progression or even transformation (10).

The success of several clinical trials in cancer prevention in high-risk populations suggests that chemoprevention is a rational and appealing strategy. 
Only few studies have been conducted on laryngeal precancerous lesions, due to limitations associated with difficulties in access and monitoring. Instead, premalignant lesions of the oral cavity represent an ideal model for study, as ready access allows easy monitoring and serial biopsies, resulting in a higher likelihood of early intervention and faster data analysis.

The aim of the present study was to determine the role of folic acid in the treatment of mild and moderate laryngeal dysplasia recurring after surgery, and compare the data obtained by laryngoscopy, measurement of serum folate levels and functional vocal assessment with the grade, instability, roughness, breathiness, asthenia and strain (GIRBAS) scale and the voice handicap index (VHI) questionnaire in patients who received folic acid with a homogeneous group of patients with dysplastic lesions who did not receive any therapy.

\section{Patients and methods}

Patients. The present study included 24 patients with persistent glottic leukoplakia, who underwent surgery at the ENT Division of the Department of Neuroscience, Reproductive and Odontostomatologic Science (University 'Federico II', Naples, Italy), between 2010 and 2015. The diagnosis was confirmed by direct microlaryngoscopy with a biopsy for histological assessment. Leukoplakia was histolocically classified as normal mucosa, hyperkeratosis without dysplasia, mild dysplasia [laryngeal intraepithelial neoplasia (LIN) I], moderate dysplasia (LIN II), severe dysplasia (LIN III), or invasive carcinoma. All the patients came from a homogeneous geographic area and included 20 men and 4 women. The mean patient age was 65 years.

The patients had been previously treated by excisional biopsy, namely type I cordotomy/cordectomy, according to the classification of the Working Committee, European Laryngological Society $(11,12)$, using 'cold' microinstruments and/or $\mathrm{CO}_{2}$ Acublade 400 Lumenis laser, Sharplan 40C and Zeiss surgical microscope during microlaryngoscopy.

A total of 18 patients underwent type I cordectomy and 6 patients underwent type II cordectomy. The histological findings included mild dysplasia in in 16 cases $(66.6 \%)$ and moderate dysplasia in the remaining 8 cases $(33.3 \%)$.

Upon joining the study, the patients were adequately informed regarding the risks of the disease and its possible progression to malignancy, as well as the high tendency of recurrence, and they all signed a consent form for the therapy. The Institutional Review Board of the University 'Federico II' approved the study protocol. Patients who were smokers or habitual alcohol consumers were excluded from the study. All patients denied using narcotics, receiving gastroesophageal reflux therapy, having any dietary deficiencies or being exposed to toxic substances by inhalation. All patients underwent the following examinations/tests: i) Ear, nose and throat examination upon enlisting, at 3 months, and at 6 months from agreeing to participate in the study. ii) Fibrolaryngoscopy with optical fibers of the airways-digestive system with the aid of video recording upon joining, at 3 months and at 6 months. The response was then classified as stable disease, when the dimensions and the appearance of the lesion remained virtually unchanged compared with the start of the study, as partial response when a reduction in the size of the lesions was observed, or as complete response when there was no evidence of residual leukoplakia. Should any lesion exhibit malignant transformation during treatment, surgical resection would have been performed; however, there were no such cases in the present study. If the lesion persisted after 6 months, the patients underwent endoscopic resection followed by a further histological assessment of the residual leukoplakia. iii) Serum folate levels upon enlisting (baseline), at 3 months and at 6 months. iv) Functional assessment of vocal emission using the GIRBAS scale and VHI questionnaire upon enrolling, at 3 months and at 6 months.

During follow-up, all the patients exhibited a tendency to relapse, with the appearance of low-degree hyperkeratosis.

The patients were randomly divided into two homogeneous groups by sex and age (groups A and B), each group including 12 patients (10 male and 2 female). In group A, 8 patients exhibited mild dysplasia and 4 had moderate dysplasia; 9 of the patients underwent type I and 3 underwent type II cordectomy with $\mathrm{CO}_{2}$ laser. The lesion was bilateral in 10 patients (83\%). In group B, 7 patients exhibited mild dysplasia and 5 had moderate dysplasia; 9 of the patients underwent type I and 3 underwent type II cordectomy with $\mathrm{CO}_{2}$ laser. The lesion was bilateral in 9 patients $(75 \%)$.

All 12 patients in group A were treated with folic acid (Folina; Schwarz Pharma, Monheim am Rhein, Germany) administered orally (15 mg every $8 \mathrm{~h}$ ) for 6 months. The 12 patients in group B received no chemoprevention treatment, but served as the control group. Prior to treatment initiation, assessment of serum folate levels was performed.

Statistical analysis. Statistical analysis was performed using the MedCalc statistical software, version 9.3.7.0 (MedCalc Software, Ostend, Belgium). Groups A and B were compared using the t-test for quantitative variables and the Wilcoxon/Mann-Whitney $\mathrm{U}$ test for non-parametric variables. A P-value of $<0.005$ was considered to indicate statistically significant differences.

\section{Results}

Response to treatment. In group $\mathrm{A}$, upon laryngoscopic examination there was a clinical improvement of up to $80 \%$ at 6 months. Specifically, 7 patients $(58 \%)$ had a complete response, with clinically evident regression of the leukoplakia, $3(25 \%)$ displayed a partial decrease in the lesions with reduced volume of the area involved, whereas 2 patients exhibited no change. As regards patients in control group B, 8 (67\%) exhibited no change, in 1 case there was spontaneous regression, and in $3(25 \%)$ there was histological progression with suspected malignant transformation that led to repeat surgery (Figs. 1 and 2). As regards smoking and drinking habits, none of the patients reported any change.

Restoration of serum folate levels. The serum folate levels at baseline $(\mathrm{t} 0)$ were low in all patients from both groups compared with normal values (range, 2.7-34 ng/ml). However, after 6 months of treatment, the difference between the two groups was statistically significant $(\mathrm{P}<0.0001$; Table I).

Functional improvement. To assess treatment efficacy, the GIRBAS scale and VHI questionnaire were used. Vocal discomfort values tended to return to normal in group A, 
Table I. Comparison of folate serum levels between patients in groups A and B.

\begin{tabular}{lcrc}
\hline & \multicolumn{2}{l}{ Folate serum levels, ng/ml } & \\
\cline { 2 - 3 } Time point, months & Group A & Group B & P-value \\
\hline 0 & $4.5 \pm 1.8$ & $4.6 \pm 1.7$ & 0.5 \\
3 & $6.5 \pm 2.0$ & $4.75 \pm 2.1$ & 0.0001 \\
6 & $6.9 \pm 1.9$ & $4.9 \pm 1.8$ & $<0.0001$ \\
\hline
\end{tabular}

Values are presented as mean \pm standard deviation.

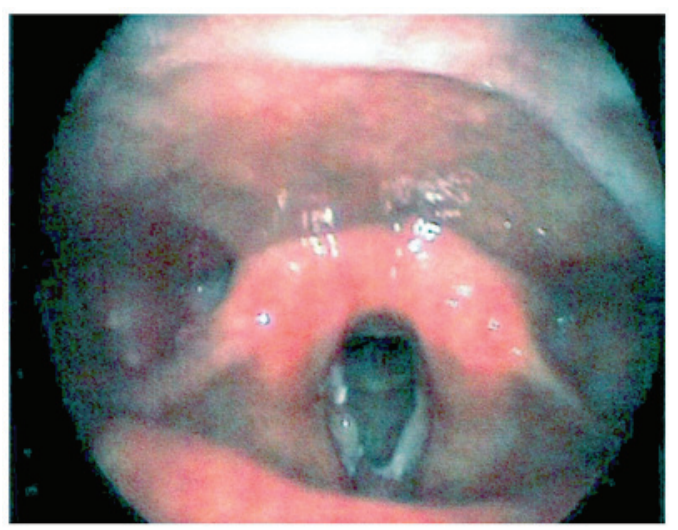

Figure 1. Laryngoscopic view in a patient with recurrence of laryngeal leukoplakia.

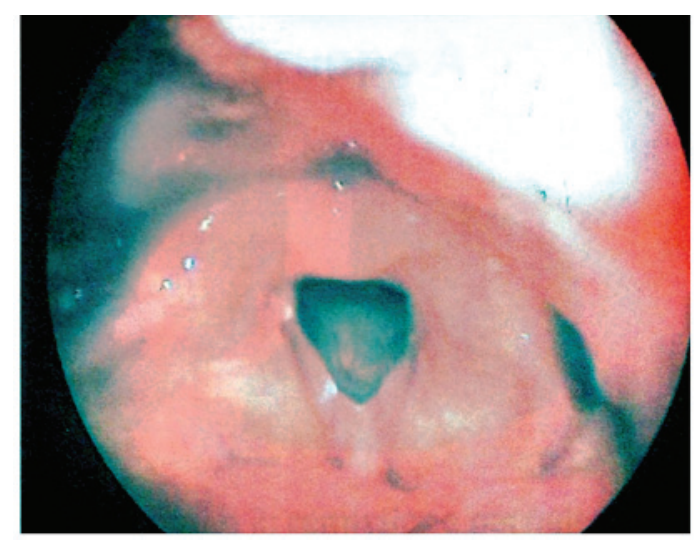

Figure 2. Laryngoscopic view of the patient in Fig. 1 after 6 months of folic acid treatment.

while they remained virtually unchanged in group B, with statistically significant differences as determined with the Wilcoxon/Mann-Whitney $\mathrm{U}$ test $(\mathrm{P}=0.004$ and $\mathrm{P}=0.001$ respectively; Figs. 3 and 4).

\section{Discussion}

A standard treatment for leukoplakia and other dysplastic lesions has not been established and the proposed approaches range from observation to endoscopic surgery (12-14). However, none of these options currently appears to effectively prevent

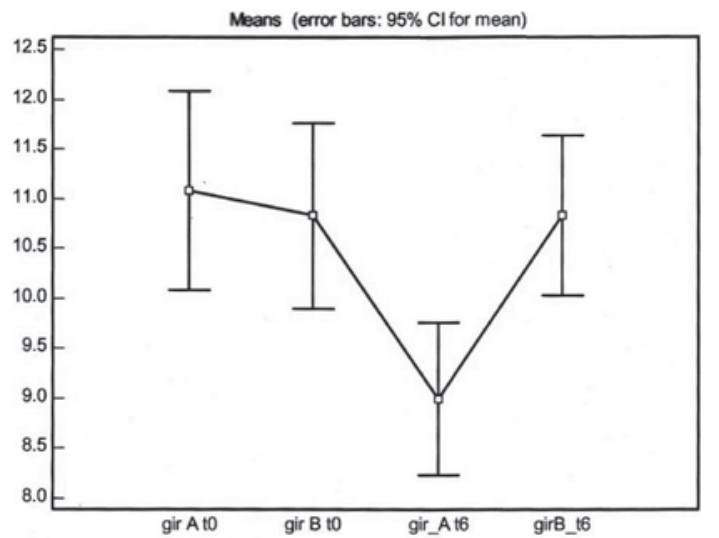

Figure 3. GIRBAS scale in groups A and B. GIRBAS scale in groups A and B at t0 and after 6 months (x-axis), mean values of 6 scale items (range $0-18$ ) in y-axis. GIRBAS, grade, instability, roughness, breathiness, asthenia and strain; CI, confidence interval.

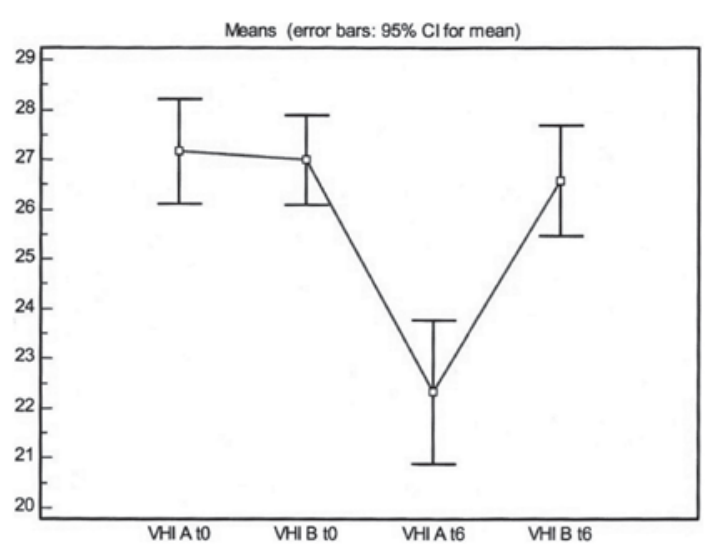

Figure 4. VHI scale in groups A and B. VHI-10 items in groups A and B at t0 and after 6 months (x-axis), mean values of 10 items (range 0-40) in y-axis. VHI, voice handicap index; CI, confidence interval

recurrence or malignant transformation (10). Thus, the larynx is a good model for the development of chemoprevention therapy. Cancer chemoprevention is the use of natural, synthetic or biological chemical agents to reverse, suppress or prevent carcinogenesis progression. The success of several clinical trials in cancer prevention in high-risk populations suggests that chemoprevention is a rational strategy. Chemoprevention may be categorized as either primary or secondary. Primary chemoprevention is directed at patients with premalignant lesions, whereas secondary chemoprevention is targeted at cancer patients who, in order to prevent disease recurrence or to prevent metachronous primary diseases, have undergone potentially curative therapy.

Folic acid plays an essential role in the stability and in the methylation and repair of chromatin. Alterations in the folate cycle were observed in patients with leukoplakia and tumors of the larynx. A decrease in the serum folate levels have been reported in subjects with dysplasia (10).

The administration of folates is associated with decreased risk of tumor of the oral cavity (4).

Various studies are evaluating the effects of folic acid on the progression of laryngeal leukoplakia; however, it has 
already been demonstrated that there is a folic acid deficiency in laryngeal neoplasia.

The majority of spontaneous clinical regressions of glottic leukoplakia occur in the first month after diagnosis (10).

In our chemoprevention protocol, the patients who suffered from recurring leukoplakia of the larynx had low serum folate levels. Folate is characterized by a low grade of toxicity and our results are encouraging, as a complete response rate of $58 \%$ was obtained in Group A patients, who were treated with folic acid and the low serum folate levels were partially corrected after 3 months and further increased after 6 months of therapy. The laryngoscopic appearance improved by $80 \%$ in the treated patients, as did vocal discomfort, which tended to resolve.

The management of head and neck cancer and laryngeal leukoplakia has become multidisciplinary. The inclusion of effective chemotherapeutic agents in the initial curative therapy of head and neck cancer has the potential to prolong survival and preserve organ function. Through well-designed and well-executed clinical trials, coupled with basic research on the biology of upper aerodigestive tract tumors, further advances in the management and prevention of these cancers may be achieved. Folate deficiency may be considered to be a factor predisposing to precancerous lesions. Dietary supplementation may prevent and inhibit the events occurring during the multistep process of carcinogenesis or the reversal of a premalignant condition (15).

\section{References}

1. Almadori G, Bussu F, Galli J, Cadoni G, Zappacosta B, Persichilli S, Minucci A and Giardina B: Serum folate and homocysteine levels in head and neck squamous cell carcinoma. Cancer 94: 1006-1011, 2002.

2. Blount $\mathrm{BC}$ and Ames BN: DNA damage in folate deficiency. Bailleres Clin Haematol 8: 461-478, 1995.

3. Eto I and Krumdieck CL: Role of vitamin B12 and folate deficiencies in carcinogenesis. Adv Exp Med Biol 206: 313-330, 1986.

4. Weinstein SJ, Gridley G, Harty LC, Diehl SR, Brown LM, Winn DM, Bravo-Otero E and Hayes RB: Folate intake, serum homocysteine and methylenetetrahydrofolate reductase (MTHFR) C677T genotype are not associated with oral cancer risk in Puerto Rico. J Nutr 132: 762-767, 2002.
5. Dobrossy L: Epidemiology of head and neck cancer: Magnitude of the problem. Cancer and Metastis Rew 24: 9-17, 2005.

6. Brugere J, Guenel P, Leclere A and Rodriguez J: Differential effects of tobacco and alcohol in cancer of the larynx, pharynx, and mouth. Cancer 57: 391-395, 1986.

7. Hail N Jr, Cortes M, Drake EN and Spallholz JE: Cancer chemoprevention: A radical perspective. Free Radic Biol Med 45: 97-110, 2008

8. Xiangwei, Wu Sherri, Patterson Ernest and Hawk MD MPH. Chemoprevention-history and general principles. Best Pract Res Clin Gastroenterol 25: 445-459, 2011.

9. Rhee JC, Khuri FR and Shin DM: Advances in chemioprevention of head and neck cancer. Oncologist 9: 302-311, 2004.

10. Almadori G, Bussu F, Galli J, Cadoni G, Zappacosta B, Persichilli S, Minucci A, Giardina B and Maurizi M: Serum levels of folate, homocysteine and vitamin B12 in head and neck squamous cell carcinoma and in laryngeal leukoplakia. Cancer 103: 284-292, 2005.

11. Remacle M, Eckel HE, Antonelli A, Brasnu D, Chevalier D, Friedrich G, Olofsson J, Rudert HH, Thumfart W, de Vincentiis M and Wustrow TP: Endoscopic cordectomy. A proposal for a classification by the Working Committee, European Laryngological Society. Eur Arch Otorhinolaryngol 257: 227-231, 2000.

12. Remacle M, Van Haverbeke C, Eckel H, Bradley P, Chevalier D, Djukic V, de Vicentiis M, Friedrich G, Olofsson J, Peretti G, et al: Proposal for revision of the European Laryngological Society classification of endoscopic cordectomies. Eur Arch Otorhinolaryngol 264: 499-504, 2007.

13. Mesolella M, Iorio B, Misso G, Luce A, Cimmino M, Iengo M, Landi M, Sperlongano P, Caraglia M and Ricciardiello F: Role of perineural invasion as a prognostic factor in laryngeal cancer. Oncol Lett 11: 2595-2598, 2016.

14. Motta S, Cesari U, Mesolella M and Motta G: Functional vocal result after $\mathrm{CO} 2$ laser endoscopic surgery for glottic tumors. J Laryngol Otol 122: 948-951, 2008.

15. Mesolella M, Iorio B, Landi M, Cimmino M, Ilardi G, Iengo $\mathrm{M}$, and Mascolo M: Overexpression of chromatin assembly factor-1/p-60 predicts biological behaviour of laryngeal carcinomas. Acta Otorhinolaryngol Ital 37: 17-24, 2017. 